\title{
How much can we learn from a single chromatographic experiment? A Bayesian perspective
}

\author{
Paweł Wiczling*, Roman Kaliszan
}

Department of Biopharmaceutics and Pharmacodynamics, Medical University of Gdańsk, Gen. J. Hallera 107, 80-416 Gdańsk, Poland

*Corresponding author: tel. ++48 58349 1493; fax ++48 58 349 3262; e-mail: wiczling@gumed.edu.pl

\section{Supplementary Material:}

The supplementary material provides:

1) Details of the model used in this work.

2) Figure $\mathrm{S} 1$. The search for the desired isocratic conditions exemplified by ketoprofen.

3) Figure S2 and Figure S3. The search for the desired methanol gradient conditions exemplified by ketoprofen and papaverine mixture. 


\section{1) Details of the model:}

The model used in this work is described as follows. Let $i$ denote the $i^{\text {th }}$ compound $(i=$ $1, \ldots, N)$ and $j$ the $j^{t h}$ chromatographic retention time for a compound $\left(j=1, \ldots, n_{i}\right)$, where $n_{i}$ is the number of observations for analyte $i$. Let $t_{R, i j}=\left\{t_{R i l}, \ldots, t_{R, i n i}\right\}$ be the $n_{i}$-vector of measurements performed for compound $i$. Let the function $f$ denotes the nonlinear structural model relating retention $t_{R, i j}$ with analyte properties and certain experimental design, $D_{i j}$. The statistical model for the observed $t_{R, i j}$ of compound $i$ under the experimental design $D_{i j}$, is given by:

$t_{R, i j}=f\left(D_{i j}, R_{i}\right)+\varepsilon_{i j}$

where $R_{i}$ is the p-vector of the individual parameters (it includes $\log k_{w}, p K_{a}, S$ and others), $D_{i j}$ is a vector of all adjustable system parameters influencing analyte retention, i.e. organic modifier content, $\mathrm{pH}$, flow rate etc., and $\varepsilon_{i j}$ is the residual error, which is assumed to be normal, with zero mean. The variance of $\varepsilon_{i j}$ may depend on the retention times predicted $f\left(D_{i j}, P_{i}\right)$ through a (known) variance model such as:

$\operatorname{var}\left(\varepsilon_{i j}\right)=\left(\sigma_{\text {add }}+\sigma_{\text {prop }} f\left(D_{i j}, R_{i}\right)\right)^{2}$

where $\sigma_{\text {add }}$ and $\sigma_{\text {prop }}$ are an additive and a proportional component of unexplained residual variability, respectively.

The standard structural model $(f)$ was used to describe the relationship between analyte retention time and mobile phase composition under isocratic and gradient conditions. It assumes a three-parameter relationship between retention factor and organic modifier content for both ionized and non-ionized form of an analyte; a linear relationship between $p K_{a}$ and organic modifier content and a sigmoidal relationship between retention factor and mobile phase $\mathrm{pH}$ :

Acids: $\int_{0}^{t_{R, i, j}-t_{0}} \frac{1}{t_{0}} \frac{1+10^{s} p H(t)_{j}-p K_{a}\left(\varphi(t)_{j}\right)_{i}}{10^{\log k_{w, N, i}-\frac{S_{1, N, S_{2} \varphi(t)_{j}}}{1+S_{2} \varphi(t)_{j}}}+10^{\left.\log k_{w, I, i}\left(1+a s_{s}^{s} p H(t)_{j}-7\right)\right)-\frac{S_{1, l, i} \varphi(t)_{j}}{1+S_{2} \varphi(t)_{j}}} 10^{s} p H(t)_{j}-p K_{a}\left(\varphi(t)_{j}\right)_{i}} d t=1$

Bases: $\int_{0}^{t_{R, i j}-t_{0}} \frac{1}{t_{0}} \frac{1+10^{p K_{a}\left(\varphi(t)_{j}\right)_{i}{ }^{s} p H(t)_{j}}}{10^{\log k_{w, N, i}-\frac{S_{1, N, i} \varphi(t)_{j}}{1+S_{2} \varphi(t)_{j}}}+10^{\log k_{w, I, i}-{ }^{-} \frac{S_{1, I, i} \varphi(t)_{j}}{1+S_{2} \varphi(t) j}} 10^{p K_{a}\left(\varphi(t)_{j}\right)_{i}-{ }_{s}^{s} p H(t)_{j}}} d t=1$

where ${ }_{s}^{s} p H(t)_{j}$ and $\varphi(t)_{j}$ are functions describing changes of mobile phase $\mathrm{pH}$ and its composition at column inlet during chromatographic run $j ; \log k_{w, N, i}$ and $\log k_{w, I, i}$ represent 
retention factors for pure water as the mobile phase of the individual ionized $(I)$ and nonionized $(N)$ forms of the analyte; $S_{l, N, i}, S_{l, I, i}, S_{2}$ are parameters which demonstrate how rapidly retention factor is changing under different organic modifier content for neutral and ionized form of analyte; $t_{0}$ denotes column hold-up time, $p K_{a}(\varphi(t) j)_{i}$ describes the $p K_{a}$ function describing the effect of different organic modifier content on analyte dissociation.

The individual (analyte-specific) parameter $\left(R_{i}\right)$ follows a multivariate normal distribution:

$R_{i}=h\left(\theta_{i}, X_{i}\right)+\eta_{R, i}$

where $\theta_{i}$ is the population vector of parameters, $X_{i}$ is a vector of covariates, $h$ is a function describing the expected value of the parameters depending on the covariates, and $\eta_{R, i}$ represents the vector of random effects for compound $i . \eta_{R, i}$ is assumed to follow a multivariate normal distribution $M V N(0, \mathbf{\Omega})$, where $\mathbf{\Omega}$ is the variance-covariance matrix of the random effects. The $\eta_{R, i}$ is a deviation of the individual estimate of a given parameter for $i^{\text {th }}$ analyte from the population mean and can also be understood as a residual error for that particular relationship. The following QSRR equations were found to describe covariate relationships:

$$
\begin{aligned}
& \log k_{w, N, i}=\theta_{\log k w}+\theta_{\log k w-\log P} \log P_{i}+\theta_{\log k w-P S A} P S A_{i}+\eta_{\log k w, N, i} \\
& S_{1, N, i}=\theta_{S N}+\theta_{S N-\log P} \log P_{i}+\theta_{\log S N-P S A} P S A_{i}+\eta_{S N, i} \\
& \log k_{w, I, i}=\log k_{w, N, i}+\theta_{\Delta \log k w}+\eta_{\log k w, I, i} \\
& S_{1, I, i}=S_{1, N, i}+\theta_{\Delta S}+\theta_{A B-\alpha} A B_{i}+\eta_{S I, i} \\
& p K_{a}\left(\varphi(t)_{j}\right)_{i}={ }_{w}^{w} p K_{a, i}+\left(\theta_{\alpha}+\theta_{A B-\alpha} A B_{i}\right) \varphi(t)_{j}+\eta_{p K a, i}
\end{aligned}
$$

In those equations $\log k_{w}$ of neutral form $\left(\log k_{w, N}\right)$ is assumed to be linearly related to drug lipophilicity $(\log P)$ and polar surface area $(P S A)$. The same type of relationship was evident for $S_{1, N}$. The retention factor of the ionized form of analyte was lower compared to the neutral form. This difference was found to be similar for acids and bases. The typical first slope parameter $\left(S_{l, I}\right)$ of the ionized form of analyte was higher for bases and lower for acids compared to the non-ionized form of analyte. It indicates that the retention factor of ionized form increases more rapidly for acids than for bases with an increase in organic 
modifier content. The second slope parameter $\left(S_{2}\right)$ was equal to 0.183 for all analyte forms. The $\log k$ of ionized form of acids slightly decreased with increasing $\mathrm{pH}$ with the slope $\alpha$ of -0.0172 . The $p K_{a}(\varphi(t))$ was proportional to the aqueous $p K_{a}$ and was dependent on organic modifier content with a slope $\alpha$ equal to 1.61 for acids and -0.365 for bases. The numerical values of all model parameters are given in the original article (Wiczling, P.; Kubik, Ł.; Kaliszan, R. Anal Chem 2015, 87. 7241-9) 
2) The search for the desired isocratic conditions exemplified by ketoprofen.

A)
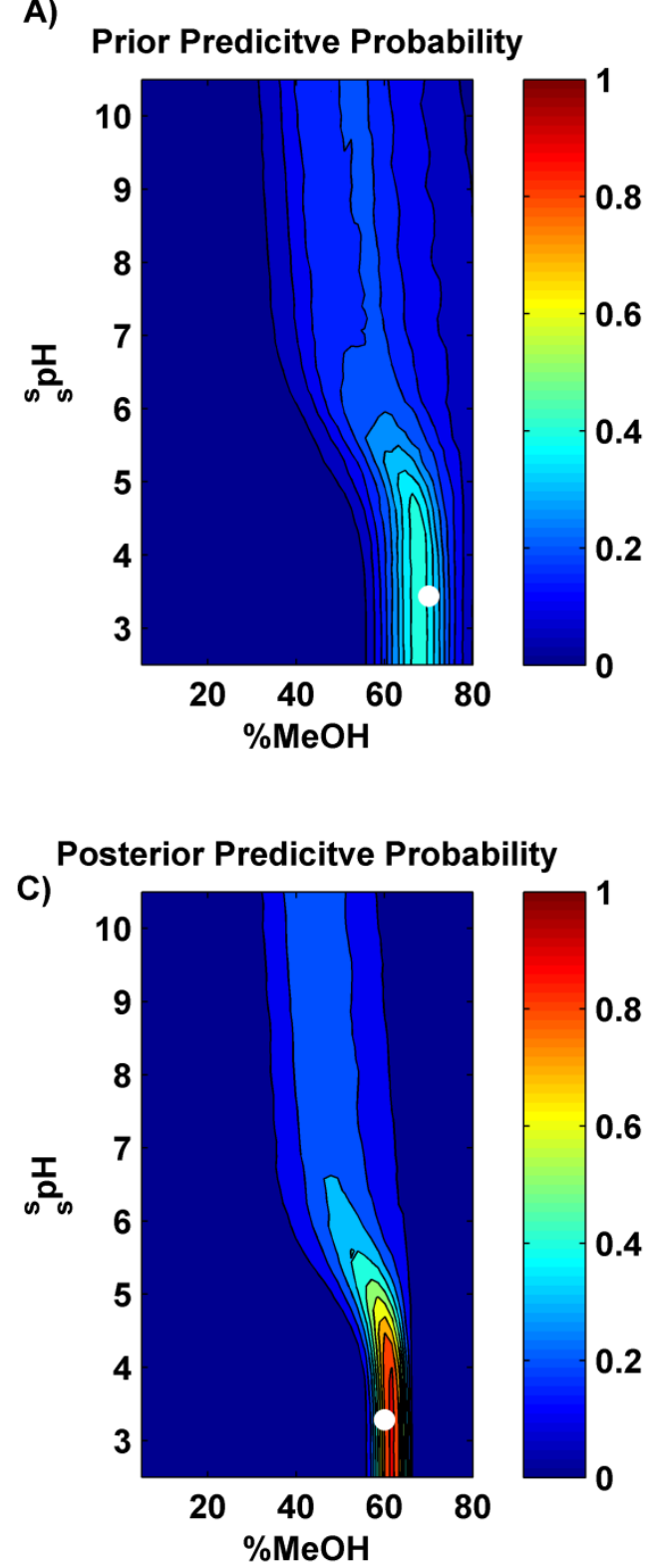

B)
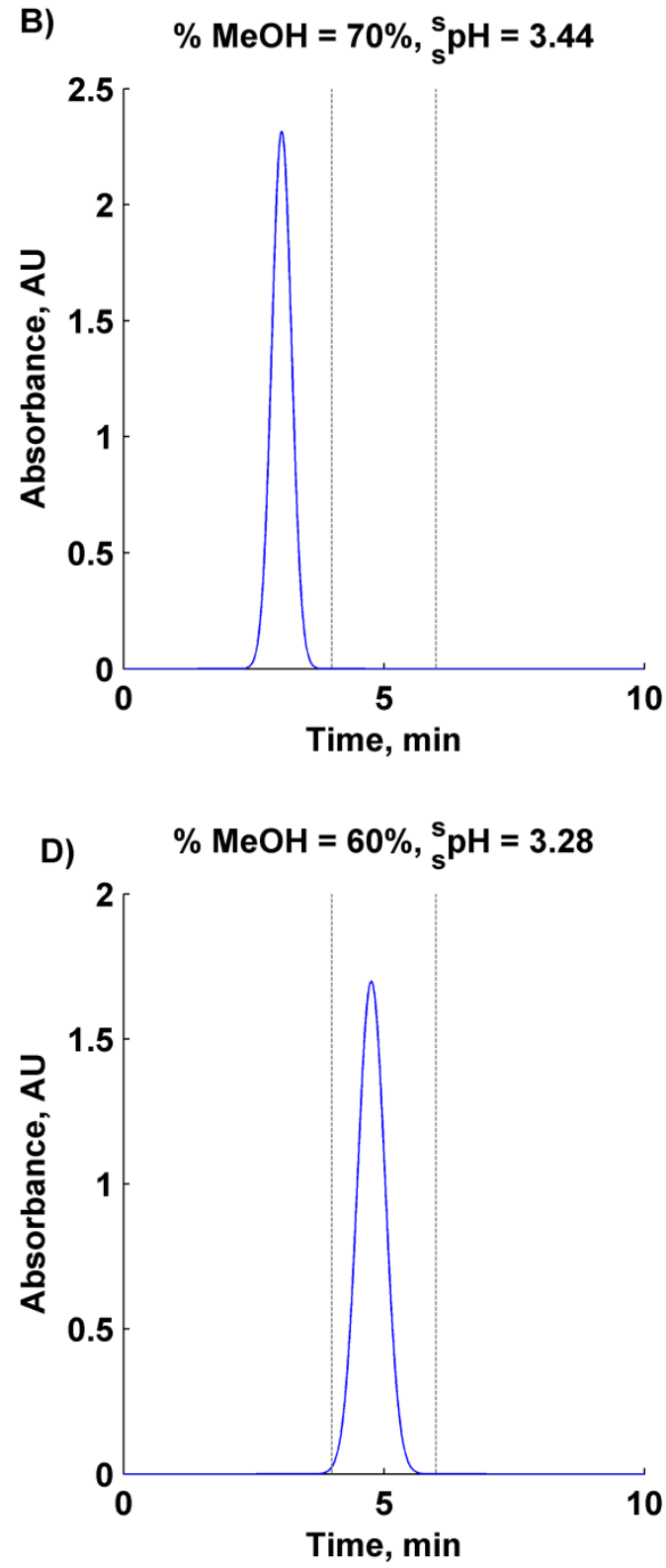

Figure S1. The sequential Bayesian approach for the optimization problem involving single analyte. The isocratic retention time of ketoprofen within a range of 4-6 min (vertical line) was sought for different combination of methanol content and mobile phase $\mathrm{pH}$. The subplot $A$ shows the prior predictive distribution. The experimental chromatogram corresponding to the maximum is given in subplot $\mathrm{B}$. Since the predictions were unsatisfactory the posterior predictive probability was obtained (subplot C). Its maximum corresponds to the desired retention time window (subplot D). This procedure allowed us to obtain a preferred chromatogram using one preliminary experiment. The white dot is the point closest to the maximum among the set of experiments that were performed. 
3) The search for the desired methanol gradient conditions (given in the figure legend) exemplified by ketoprofen and papaverine mixture. The details about the experiments are given in the following article (Wiczling, P.; Kaliszan, R. $J$ Chromatogr A 2010, 1217.3375-81).
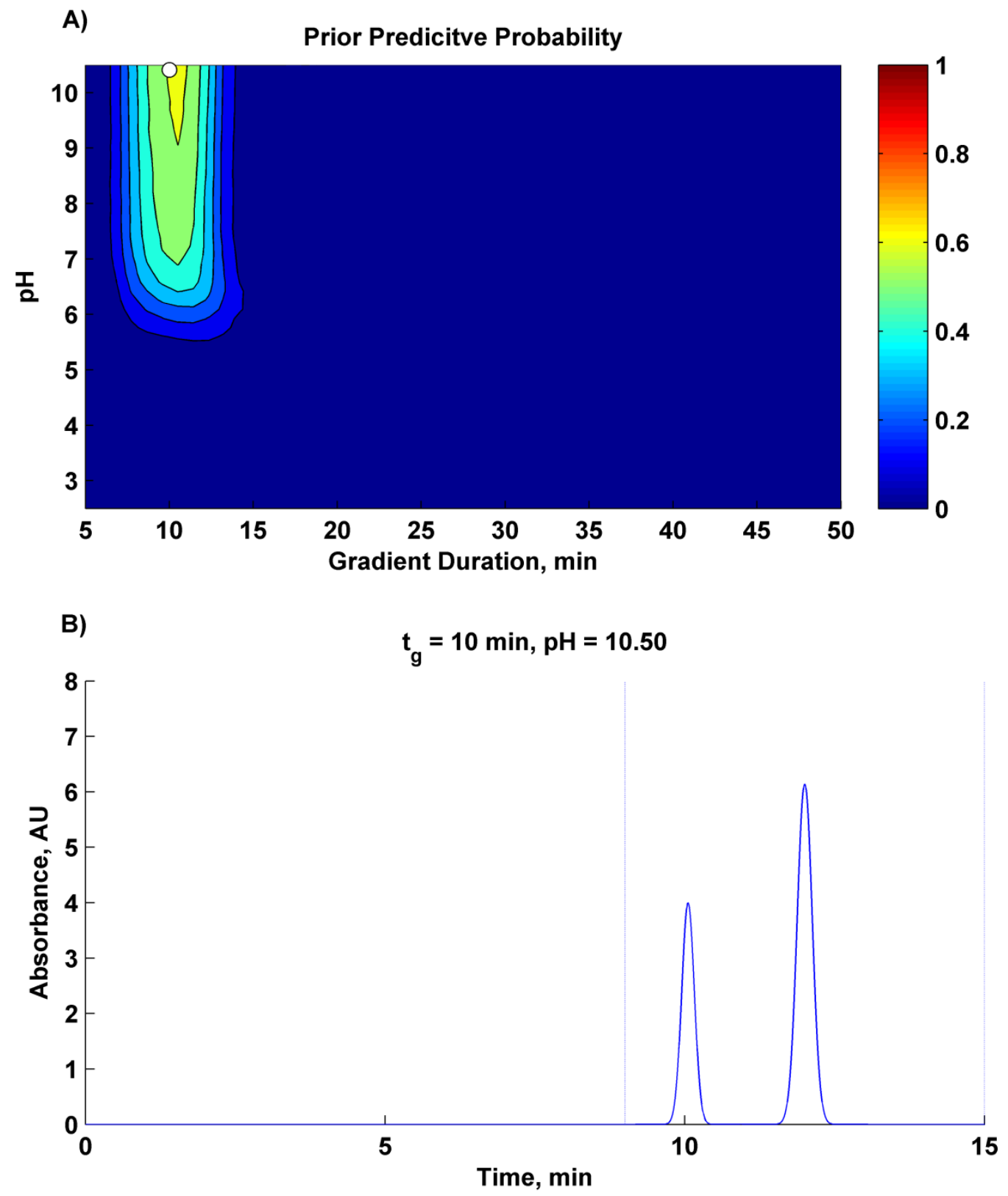

Figure S2. The sequential Bayesian approach for the optimization problem involving two analytes. The absolute gradient retention time difference between ketoprofen and papaverine larger than 1 min, retention time within a range of 9-15 $\mathrm{min}$ for both analyte, and retention time for ketoprofen lower than for papaverine was sought for a different combinations of gradient durations and mobile phase $\mathrm{pH}$ (at gradient start). The subplot $\mathrm{A}$ shows the prior predictive distribution. The experimental chromatogram corresponding to the maximum is given in subplot $B$. In this case a preferred chromatogram was obtained without performing preliminary experiments. The white dot is the point closest to the maximum among the set of experiments that were performed. 
A) Prior Predicitve Probability

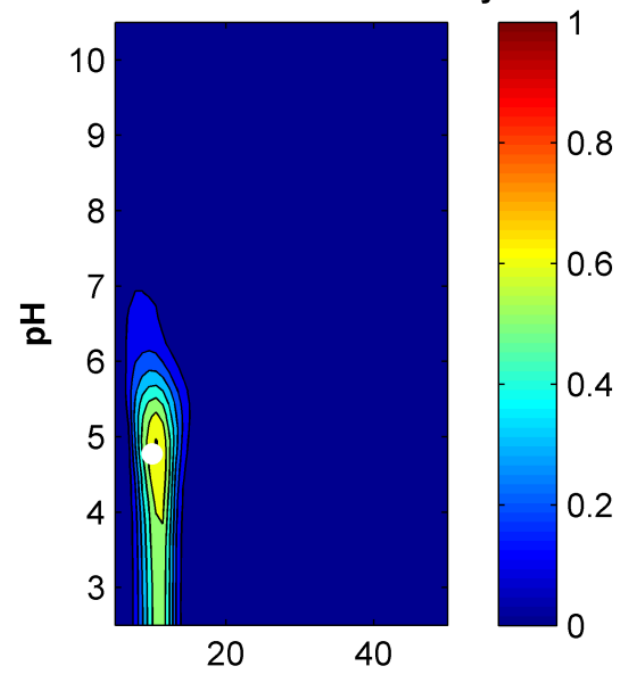

Gradient Duration, min

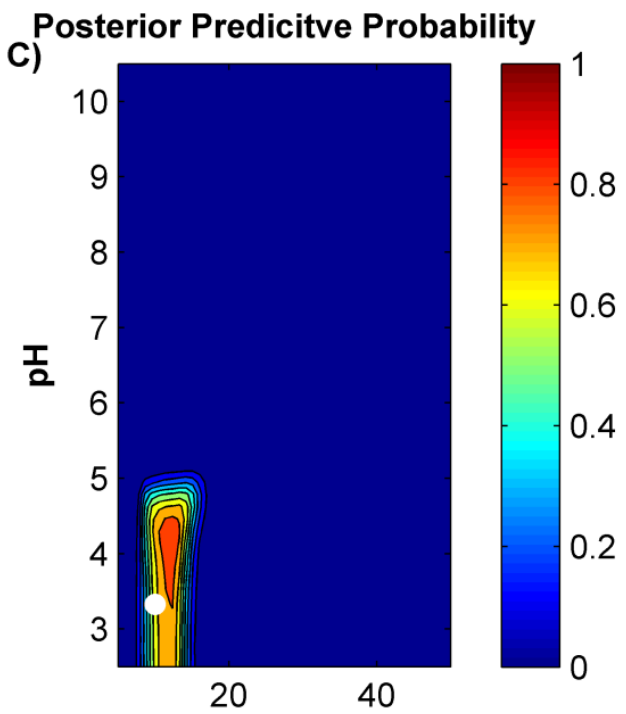

Gradient Duration, min

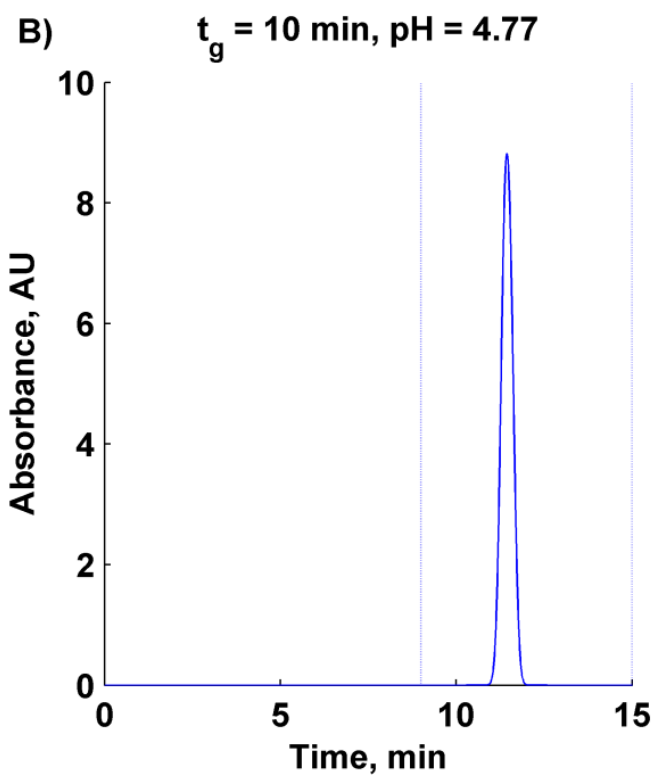

D)

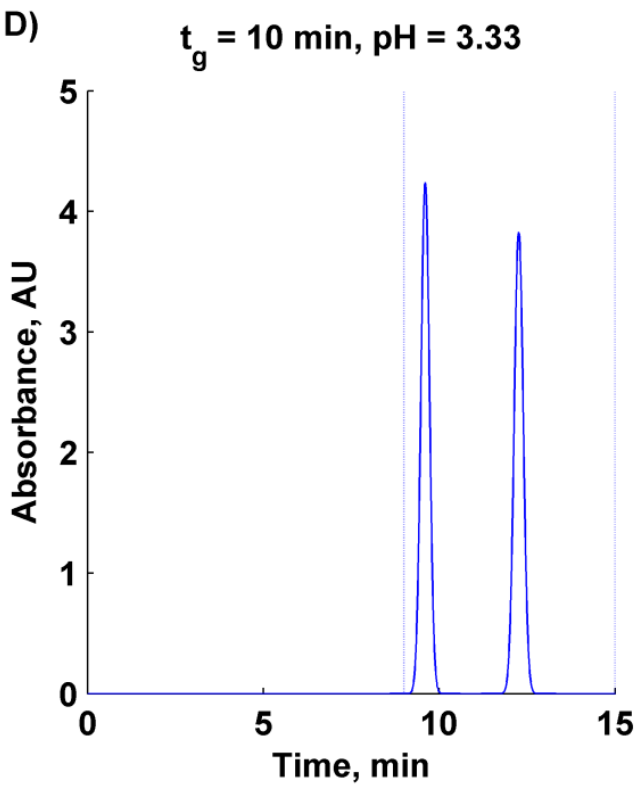

Figure S3. The sequential Bayesian approach for the optimization problem involving two analytes. The absolute gradient retention time difference between ketoprofen and papaverine larger than 1 min, retention time within a range of 9-15 $\mathrm{min}$ for both analyte, and retention time for papaverine lower than for ketoprofen was sought for a different combinations of gradient durations and mobile phase $\mathrm{pH}$ (at gradient start). The subplot $\mathrm{A}$ shows the prior predictive distribution. The experimental chromatogram corresponding to the maximum is given in subplot B. Since the predictions were unsatisfactory (peak overlap) the posterior predictive probability was obtained (subplot C). Its maximum corresponds to the desired separation (subplot $\mathrm{D}$ ). This procedure allowed us to obtain a preferred chromatogram using one preliminary experiment. The white dot is the point closest to the maximum among the set of experiments that were performed. 